\title{
Factores de riesgo para la adicción en los adolescentes
}

\section{Risk factors for addiction in adolescents}

\section{Fact riesg adicc adolentes}

Mercedes María Lucas Choéz Mg ${ }^{(1)}$

Martha Saida Quiroz Figueroa Mg (2)

Cristhian Jonathan Cedeño Alava Mg ${ }^{(3)}$

(1) Universidad Estatal del Sur de Manabí, Facultad de Ciencia de la Salud, Jipijapa - Manabí, mercedeslucas_ch86@hotmail.com

(2) Universidad Estatal del Sur de Manabí, Facultad de Ciencia de la Salud, Jipijapa - Manabí marthaquiroz85@hotmail.com

(3) Hospital Jipijapa, Jipijapa - Manabí,_lcdocrisjo@gmail.com

Contacto: mercedeslucas_ch86@hotmail.com

\section{Receptado: 20/09/2017}

\section{Aceptado: 14/11/2017}

\section{Resumen}

La presente investigación se dio ante la evidencia de la problemática actual existente en nuestra sociedad de indagar las razones principales que incitan a los adolescentes a involucrarse en el uso y consumo de drogas siendo el objeto de estudio las adicciones escolares teniendo como objetivo general determinar factores de riesgos que conllevan a los estudiantes a adicciones en etapa escolar, utilizando una muestra aleatoria de 175 estudiantes del primer año de bachillerato, para el desarrollo de la investigación se utilizó el método analítico y sintético-deductivo, en la recolección y sistematización de la información se emplearon encuestas y entrevistas dando como resultados más relevantes los problemas familiares que engloban el $70 \%$ afectando a los jóvenes y que predisponen a que los estudiantes se involucren con estas sustancias ilícitas, siendo la heroína comúnmente denominada "H" con el $67 \%$ la droga más consumida, éstos resultados y la importancia de los educandos en obtener conocimientos con el 94\% sobre estas adicciones se estableció estrategias preventivas tanto en el ámbito educativo como familiar para bajar el índice de adicciones escolares por lo cual se propuso un programa de participación estudiantil para la prevención integral del uso y consumo de drogas en los estudiantes de la Unidad Educativa Fiscal Alejo Lascano.

Palabras claves: Adolescencia, consecuencias, drogas, dependencia, prevención. 


\begin{abstract}
The present investigation was given to the evidence of current problems in our society to investigate the main reasons that encourage adolescents to become involved in drug use consumption, the object of study is school addiction with the general objective of determining factors of risks that lead students to addictions at school, using a random simple of 175 students of the first year of high school, for the development of research was used the analytic and synthetic-deductive method, in the collection and sytematization of information used surveys and interviews, were used giving as more relevant results the family problems that involve $70 \%$ affecting the young and predispose to that the students are envolved with these ilicit substances, heroin being commonly called " $\mathrm{H}$ " with $67 \%$ the most consumed drug, these results and the importance of students in obtaining knowledge with $94 \%$ on these addictionss established preventive strategies both in the educational and family to lower the rate of school addictions for which proposed a program of student participation for the comprehensive prevention of drug use and consumption in students of the Educational Unit Prosecutor Alejo Lascano.
\end{abstract}

Keywords: Adolescence, consequences, drugs, dependence, prevention.

\title{
Introducción
}

La adolescencia es la etapa o período que llega después de la infancia y que comprende desde la pubertad hasta el completo desarrollo del cuerpo. En otras palabras la adolescencia, es la transformación del infante antes de llegar a la adultez; todos estos sucesos son etapas de la vida por el cual una persona debe pasar para convertirse en adulto, en el cual va a ver un conjunto de cambios tanto físicos como sociales. Comprende 3 etapas que varían en su duración: Adolescencia precoz comprendida entre los 12 y 13 años de edad, adolescencia media entre los 14 y 16 años de edad y adolescencia tardía entre los 17 y 21 años de edad. Dentro de los cambios fisiológicos existen modificaciones en el desarrollo físico en el que el adolescente va a estar inmerso en un mundo de confusiones y admiración sobre lo que sucede con su cuerpo, otros de los desarrollos a evolucionar será el intelectual, emocional y social que le ayudarán a forjar una identidad propia. No todos los adolescentes se desarrollan de la misma manera, al final del período de la adolescencia el desarrollo intelectual, emocional y social van a alcanzar su desarrollo físico completo (1).

La adolescencia es un fenómeno biológico, social y cultural. Desde tiempos más pasados se han venido registrando quejas sobre los adolescentes y se prueba que esta etapa es compleja, enredada y 
llena de riesgos, debido a que existen cambios físicos, emocionales, psicológicos y sociales en los adolescentes, en ellos suelen presentarse desconocimiento o curiosidades con sus nuevos cambios personales por lo que les motiva a buscar de respuesta tomando muchas veces decisiones erróneas, tornándose de conductas riesgosas para su vida (2).

Dentro de uno de los principales cambios que se experimenta en los adolescentes está el desarrollo del pensamiento abstracto y sensato, la determinación de la identidad sexual, fortalecimiento de amistades, las experimentaciones con nueva sensaciones que se pueden convertir muchas veces en adicciones. "Según la Organización Mundial de la Salud (OMS) la adicción es una enfermedad física y psicoemocional que crea una dependencia o necesidad hacia una sustancia, actividad o relación" (3). Una persona que se sienta dependiente de algo se puede decir que es una persona adicta por lo que existen muchos factores predisponentes que conllevan a presentar este tipo de problema tanto a nivel personal y social.

La etapa de la adolescencia se caracteriza por una temprana tentativa de nuevas experiencias y sensaciones. El consumo de sustancias psicoactivas a temprana edad es un problema de salud pública y social muy complicada y multifactorial, debido a sus causas, consecuencias e implicaciones en los adolescentes, por tanto se puede observar desde diferentes perspectivas; los múltiples elementos implicados determinan muchos aspectos que deberían ser considerados para poder entender y abordar de manera exhaustiva esta problemática social. Hay que tener presente que en nuestra sociedad existen diferentes factores que invitan a un estilo de vida consumista, marcado por la búsqueda de nuevas sensaciones, el hedonismo, la individualidad, el placer inmediato sin tener en consideración las consecuencias a medio o largo plazo, lo que va a propiciar también el inicio en las adicciones en adolescentes; los peligros y perjuicios relacionados a adicciones en esta etapa varían de acuerdo a cada sustancia, es por ello que hay que tener en cuenta los factores personales, el nivel de motivación, la experiencia y las propiedades específicas de adicciones en adolescentes. Ante estas influencias tanto la familia como el sector educativo tienen un papel primordial, de ahí la importancia de la prevención de estos ámbitos (4).

El mundo ha avanzado mucho en tema de medicina, tecnología y otras ciencias del saber humano, por lo que no todo ha sido cambios positivos, siendo la creación de nuevas drogas o sustancias adictivas uno de los avances negativos, con los que se puede corromper a las nuevas generaciones. Existe una variedad de factores tanto de protección como de riesgo que conllevan al consumo de sustancias psicoactivas y psicotrópicas. Al hablar de drogas en general, la familia se muestra como pilar fundamental en la prevención del consumo, así mismo el sistema educativo también puede 
favorecer el correcto ajuste psicosocial del menor y tratándose de un estilo educativo indulgente puede servir como factor de protección para evitar el consumo. Cuando se relacionan con el consumo de otros tipos de drogas, el estrés, factores familiares, factores individuales como el autoestima o el bajo autocontrol y rasgos de cambios de personalidad como por ejemplo la impulsividad, entre otras, todos estos aspectos se destacan dentro de los factores predisponentes para el consumo de sustancias psicoactivas en adolescentes (5).

La adicción a sustancias es un fenómeno complejo, determinado por un avance que va desde el uso experimental, al uso social, el abuso y la dependencia. El adolescente a lo largo de este camino experimenta momentáneas e intensas sensaciones de satisfacción, encontrando un medio rápido para escapar de situaciones de emocionales difíciles y vive una falsa ilusión de autoridad ante las labores del desarrollo psicosocial propias de la edad. "Una adicción es una enfermedad física y emocional" (6), actúa también como una dependencia o necesidad hacia cualquier sustancia o actividad suscitada especialmente por el agrado que esta origina a la persona. El adolescente en el transcurso de la adicción tiene insuficiente confianza en su experiencia para enfrentar al mundo, sin los químicos por lo que prefieren esquivar la realidad de cualquier problema que se les presente o al contrario entrar en un mundo de fantasías donde se sienten libres de problemas a través del consumo de sustancias psicoactivas.

Las drogas en los últimos años no solamente se ha convertido en un asunto de seguridad nacional, sino que también su difusión se cierne como una incuestionable inminencia de salud pública y social. Además se trata de un factor excepcionalmente dinámico de muchos aspectos entre ellas la iniciación de nuevas sustancias cada vez más viables, más potentes y más adictivas. La etapa de la adolescencia constituye una alta vulnerabilidad al inicio del consumo de drogas; ellos piensan que consumir que consumir drogas les ayudará a pensar mejor, ser más populares o ser más activos. Otros consideran que estar dentro de grupos consumidores no será afectado por el bullyng o que les ayudará a escapar de problemas personales. Uno de los factores principales que puede presentarse como riesgo para el adolescente es el factor familiar en cuanto hay ausencia de normas familiares sobre el uso de drogas, los conflictos entre los padres y el adolescente o muchas veces el consumo de alcohol entre padres. No cabe duda que los comportamientos más problemáticos de los jóvenes en la actualidad son el consumo de alcohol y otras sustancias psicoactivas, no en vano la adolescencia constituye la etapa de mayor riesgo en el inicio de conductas de consumo (7).

La investigación publicada en The American Journal of Psychiatry determinó que "los circuitos cerebrales implicados en el desarrollo de las aficiones sufren profundos cambios durante la 
adolescencia, lo que incita a las personas de estas edades a tener nuevas experiencias" (8), es decir, esta investigación trata de demostrar que las adicciones juveniles deben ser miradas como desórdenes del desarrollo neuronal o psicológico, ya que a esta edad las regiones cerebrales que rigen los impulsos no están completamente formadas, lo que significa que los adolescentes se ven impulsados a acciones compulsivas y conductas arriesgadas en sus vidas.

Estudios realizados a nivel mundial se determinó un extenso grado de hábitos de consumo de alcohol en cinco países de Sudamérica y el Caribe, más del 75\% de los estudiantes secundarios han consumido alcohol en algún momento de su vida. Existe una amplia gama de rangos de prevalencia del consumo de alcohol entre los estudiantes secundarios en Sudamérica, desde menos de un $20 \%$ a casi un $70 \%$. Ecuador, El Salvador y Venezuela muestran las tasas de consumo más bajas en un $20 \%$ o inferiores (9).

El consumo de drogas es un problema de salud pública muy severo que afecta a todos los países del mundo. En 1988 se realizó el primer estudio confiable concerniente con el uso de sustancias psicoactivas en el Ecuador dando por iniciado la campaña "antidrogas" vinculada a los convenios internacionales, con el fin de contribuir a erradicar esta problemática, este proyecto que se realizó en unión a otros países fue con el propósito de adelantar en el desarrollo de mejores metodologías para evaluar el consumo, abuso de drogas y factores de riesgo asociados, apoyando en el intercambio de experiencias entre los países participantes.

Otro estudio determinó que en Ecuador, las provincias donde es más visible la presencia de estupefacientes son la región Costa y Amazonía. En las zonas urbanas este porcentaje supera (34\%) al área rural (18\%). En la Costa, el 38\% de los adolescentes habría visto usar drogas a algún estudiante, una cifra mayor que en otras regiones (10).

Se podría refutar que el origen de consumo de drogas se sitúa en la infancia temprana, iniciándose con el consumo de una sola sustancia y a medida que aumenta la implicación, el consumo se expande tanto en frecuencia como en cantidad y avanza a múltiples sustancias. Habitualmente los jóvenes adolescentes inician con el consumo de bebidas alcohólicas y el uso de cigarrillos porque son la forma más preponderante y ampliamente disponible de conseguirlo. Puesto que son numerosas las vías que acarrean a la iniciación del consumo en un primer momento y más tarde a su estabilidad resulta más preciso intentar conceptualizar el consumo de drogas desde un aspecto centrado en los factores de riesgo, es decir cuánto más factores se atesoren mayor será la posibilidad de que un adolescente se convierta en un consumidor ocasional y habitual. 
Las bebidas alcohólicas son la primera droga que ingieren los adolescentes, su consumo en la adolescencia es notablemente nocivo para la salud y más aún si es consumido antes de los 15 años de edad tienen más probabilidad de volverse adictos en algún momento de sus vidas. Es por ello que se debe procurar manejar este tipo de problema con rapidez para evitar secuelas a largo plazo en los adolescentes (12).

Según la Organización de los Estados Americanos (OEA) las drogas impactan y cambian el cerebro. Se podría decir que las estructuras cerebrales tienen una amplia relación con las conductas asociadas al consumo de drogas, además de los efectos a corto y largo plazo que pueden ocasionar al consumir sustancias psicoactivas y la intervención de factores medioambientales. Sin embargo lo que lleva a un individuo a involucrarse en el consumo de drogas y a desarrollar dependencia en ellas es porque a nivel cerebral implica una interacción entre el cerebro y una serie de determinantes biológicos, psicológicos y sociales del entorno de la persona. La dependencia se caracteriza por el uso compulsivo de drogas, a pesar de saber de las consecuencias negativas que conlleva su uso. Este comportamiento es considerado como una "mala decisión" que el sujeto toma de forma voluntaria (13).

Si bien la probabilidad de consumir una droga en particular aumenta con el consumo de cualquier otra, algunas variables asociadas al consumo de drogas no pueden modificarse mediante la intervención. Sin embargo, una serie de factores predisponentes al consumo son objetivos potenciales para la intervención. En vista de lo que conocemos acerca de la sucesión y avance progresivo del consumo, lo más natural es que los programas de prevención apunten a las sustancias que surgen al inicio de esta progresión, esto propone la posibilidad de obstaculizar el proceso progresivo desde estas sustancias a otras formas de consumo, una segunda razón para centrarse en este tipo de consumo de drogas es que dichas sustancias son responsables de las enfermedades y muertes relacionadas con esta problemática cada año (14).

El consumo cotidiano de drogas determina constantes cambios en el sistema nervioso central. En sí, un solo factor no puede determinar que se desarrolle dependencia a las drogas, sin embargo existen múltiples factores de riesgo asociados que han sido identificados científicamente que conllevan al desarrollo de la adicción. Entre ellos, uno de los principales es la edad del primer consumo, puesto que el cerebro en niños y adolescentes aún está en proceso de desarrollo, por lo tanto el consumo de drogas en esta etapa puede ocasionar secuelas significativas a largo plazo (15).

A consideración de los distintos factores relacionados al inicio y a la continuidad del consumo de drogas se sugiere múltiples objetivos potenciales para la intervención de diversos programas de 
prevención, teniendo en cuenta la multiplicidad de los factores predisponentes que aquejan al adolescente.

Razón por la cual se define como problema principal factores de riesgos que conllevan a adicciones escolares en los adolescentes de la Unidad Educativa Fiscal Alejo Lascano.

Se establece como objeto de estudio: Las adiciones escolares puesto que se determina como una dependencia o necesidad hacia una actividad o sustancia, ya que el consumo de esta sustancia en la adolescencia especialmente si es en grandes y múltiples cantidades puede alterar el desarrollo a nivel cerebral afectando tanto las estructuras como su fisiología normal; y por ende originar problemas cognitivos o de aprendizaje haciéndolo más vulnerable a la adicción.

Considerando los aspectos señalados el objetivo general del presente estudio consistió en determinar factores de riesgo que conllevan a los adolescentes a adicciones en etapa escolar. Ya que los factores desencadenantes principales como el facilismo de acceder a las sustancias psicotrópicas y psicoactivas, problemas intrafamiliares o simplemente la curiosidad conllevan a consecuencias fatales en los adolescentes a largo plazo afectando no solo a él sino a su entorno.

En este sentido busca mejorar las condiciones de salud mediante la promoción de estilos de vida saludables, campañas de concienciación, la educación y la investigación. Para desarrollar esta investigación se destacan los siguientes objetivos específicos.

$>$ Analizar desde el punto de vista histórico las adicciones en etapa escolar.

$>$ Establecer factores de riesgo determinantes en la adición escolar.

> Determinar estadísticamente las principales adicciones que afectan a los estudiantes de la Unidad Educativa Fiscal Alejo Lascano.

$>$ Analizar las repercusiones de las adicciones en la salud de los adolescentes.

> Aplicar programa de participación estudiantil para la prevención integral del uso y consumo de drogas en los estudiantes de la Unidad Educativa Fiscal Alejo Lascano.

\section{Materiales y métodos}

El presente estudio se direccionó bajo los siguientes métodos aplicados: analítico-sintético, inductivodeductivo. La población objeto de estudio estuvo conformado por 505 estudiantes según el registro de secretaria de la Unidad Educativa Fiscal Alejo Lascano del cantón Jipijapa. Aplicando fórmula de muestreo probabilístico, se obtuvo un total 175 alumnos del primer año de bachillerato sección diurna seleccionado aleatoriamente, presentando un margen de error del $6 \%$. 
De acuerdo a la obtención de la información se aplicó entre los métodos empíricos: entrevistas realizadas a directivos de la institución involucrada al Lic. Milton Cañarte Cantos (Psicólogo educacional), Lic. Genny Ponce Cedeño y Lic. Luz María Ponce Gamboa integrantes del Departamento de Consejería estudiantil de la Unidad Educativa Fiscal Alejo Lascano del cantón Jipijapa, donde a través de la conceptualización: de como diagnostican si un estudiante presenta algún tipo de adicciones, manifestaron que se evidencia por los diversos comportamientos, entre ellos la agresividad y el bajo rendimiento académico, haciendo énfasis a través de investigaciones de los involucrados sobre la causas del comportamiento presentado. Dentro de las pautas para resolver esta problemática referían que se brinda ayuda psicológica y de orientación para mejorar el cambio de conducta y prevenir cualquier tipo adicción.

Se revisaron registros de expedientes de fichas de los estudiantes, destacando el total de 21 casos de jóvenes con antecedentes por consumos de drogas reportados en el año 2016, donde el mayor número de consumidores se encuentran los adolescentes del primer año de bachillerato con un total de 9 casos reportados, destacando la confidencialidad de los datos registrados y manteniendo el anonimato de los involucrados.

\section{Resultados}

En la encuesta se contó con la participación de 175 estudiantes del primer año de bachillerato sección diurna de la Unidad Educativa Fiscal Alejo Lascano del cantón Jipijapa donde se establece los principales resultados:

En relación a los diferentes tipos de adicciones que el adolescente está predispuesto a involucrarse se destaca que el $65 \%$ tiene conocimiento que el tipo de adicción más relevante en los adolescentes es el consumo de drogas, el $20 \%$ las redes sociales, en un 8,5\% los juegos tecnológicos, uso del internet u otros con un $3 \%$, es evidente que los estudiantes están conscientes que ésta es una problemática social que está atacando a los adolescentes, afectando su vínculo social y familiar. (Ver tabla \# 1)

La población más vulnerable en adiciones con un $70 \%$ se encuentran los adolescentes, el $15 \%$ infancia y adultez, estos resultados nos indican que la adolescencia es la etapa de mayor vulnerabilidad de caer en adicciones en el sentido de descubrir nuevas experiencias se involucran en adiciones que repercute la salud de los jóvenes. (Ver tabla \#2)

Entre los factores que involucran adolescentes a las drogas se encuentran los problemas familiares con el $70 \%$, curiosidad $15 \%$ y malas influencias con $14 \%$. De acuerdo a estos resultados se debe 
estimar que se debe tratar de conversar con los padres de familia para darles a conocer que en vez de que la familia se convierta en un factor de riesgo se lo fortalezca como un factor protector para evitar esta problemática, ya que es el mayor factor predisponente. (Ver tabla \#3)

Entre los tipos de drogas más consumidas por los adolescentes en la actualidad se encuentran la Heroína comúnmente conocida como "H" con el 67,4\%, seguido de las bebidas alcohólicas con el 17\%, Cannabis (marihuana) 10\% y cocaína 5\%, estos datos nos indican que la mayoría de estudiantes de esta institución conocen las diferentes sustancias psicoactivas que se encuentran en la sociedad hoy en día. (Ver tabla \#6)

Con relación a los temas educativos sobre las adicciones en la institución con talleres pedagógicos el 94\% respondió que sí, mientras que un 6\% respondió que no, con estos resultados se puede comprobar que la mayoría de estudiantes apoyan la idea de conocer más sobre esta problemática que está afectando a los adolescentes en la actualidad y así poder proponer una estrategia preventiva para evitar o disminuir este problema social. (Ver tabla \#10)

\section{Discusión}

En este trabajo se pretendió determinar los factores de riesgo que conlleva a los adolescentes que adopten adicciones como el consumo de drogas, redes sociales y otros. Los resultados mostraron inicialmente que la principal adicción que tienen los adolescentes es el consumo de drogas que concuerda con un estudio realizado en el año 2013 donde analizaron el consumo de drogas en los adolescentes obteniendo un 48\%; porcentaje no muy alejado de nuestros resultandos donde también da una cifra mayor en el consumo de sustancia psicoactivas durante esta etapa.

Referente al porcentaje correspondiente al uso de redes sociales e internet, se tiene algunas controversias, puesto que se consideran que esto no podría definirse como adicción, sin embargo, una vez que se establece las consecuencias de su uso irregular y prolongado presenta disfuncionalidades sociales que muchas veces a los adolescentes les resulta difícil o imposible estar fuera de internet; demostrando comportamiento desadaptativo y que requiere atención porque va en aumento la tecnología que en ocasiones se convierte en algo indispensable.

En cuanto a los factores que involucran el consumo de drogas varias estudios indican que los problemas familiar son las principales causas que conllevan al uso y abuso de sustancias psicoactivas especialmente cuanto mayor es el disgusto de la familiar. En el caso del consumo del tipo de droga encontramos que "H", alcohol y cannabis son las más utilizadas resultado que concuerda con estudios 
realizados en el año 2014 en estudiantes españoles, resultó significativa en cuanto a la cantidad consumida era el cannabis ya que está más asociado a factores de mayor riesgo familiar y una vez que se ha iniciado el consumo éste se vuelve más problemático, además de que está asociado a una edad media mayor de consumo.

Para futuras investigaciones sería oportuno distinguir entre diversas frecuencias de consumo, y no únicamente a los que declaran el consumo de drogas en alguna ocasión o habitualmente, algo que se considera una limitación del estudio. Hacer un análisis más amplio delimitando los grupos asociados al consumo de forma más específica, diferenciando, por ejemplo, entre consumo esporádico y consumo habitual.

Esto permitiría un nivel más profundo de análisis acerca de la influencia de la impulsividad en el consumo de sustancias psicoactivas, pudiendo encontrar diferencias dentro del grupo de sujetos que sí consumen, atendiendo a los distintos patrones de consumo.

Finalmente, de cara a la aplicación práctica de los datos que aporta el estudio, es de destacar la necesidad de contar con información actualizada, en la medida en que, el consumo drogas por los adolescentes, se trata de un fenómeno cambiante y en continua evolución. En esta línea, han de orientarse las investigaciones que analizan otros factores que pudieran estar relacionados con el inicio, mantenimiento del consumo en adolescentes, precisamente, para el diseño de recursos eficaces para su prevención.

\section{Conclusiones}

$\checkmark$ Analizando el punto de vista histórico a través de las investigaciones realizadas a nivel mundial y nacional este problema de las adicciones ha venido incrementándose cada vez más involucrando ahora a los adolescentes que por su vulnerabilidad son presa fácil en caer en diferentes tipos de adicciones siendo la más predisponente en la actualidad el consumo de sustancias sujetas a fiscalización.

$\checkmark$ Entre los factores de riesgo más destacados que conllevan a un adolescente a involucrarse en el uso y consumo de drogas fueron los problemas familiares seguido del factor curiosidad que predisponen a esta problemática en la actualidad.

$\checkmark$ A través de fichas de registros del Departamento de Consejería Estudiantil se pudo determinar estadísticamente que 21 estudiantes tienen antecedentes de uso y consumo de drogas y según resultados obtenidos a través de la encuesta y entrevista aplicada al primer año de bachillerato y 
personal de la institución educativa refieren que entre las principales adicciones que afectan a los estudiantes ha sido el consumo de sustancias sujetas a fiscalización.

$\checkmark$ En lo concerniente a salud las repercusiones más frecuentes que se han presentado en los estudiantes consumidores son a nivel psicológico en donde el adolescente consumista tiende a tener déficit de atención, afectación de la memoria, trastornos en el aprendizaje (lo que origina en fracaso escolar), presenta conductas no acorde a lo normal, ausentismo de la realidad, a nivel físico va a existir un déficit nutricional, aceleración del ritmo cardíaco, dilatación de las pupilas, entre otras, en el cual los adolescentes que presentan una adicción son derivados al Centro de salud Jipijapa para recibir su tratamiento correspondiente.

$\checkmark$ Se elaboró un programa de participación estudiantil conformado por un comité del mismo estudiantado en el cual se empleará una guía educativa para reforzar los conocimientos sobre esta problemática afectante en los estudiantes adolescentes para prevenir y disminuir el uso y consumo de drogas en esta institución educativa.

\section{Bibliografía}

1. Pérez J, Merino M. Definición.de. [Online].; 2012 [cited 2017 Enero 16. Available from: http://definicion.de/adolescencia/.

2. Hidalgo M, Júdez J. Adolescencia de alto riesgo. Consumo de drogas y conductas delictivas. Pediatr Integral. 2012; XI(10).

3. Organización Mundial de la Salud (OMS). Adicción. Organización Mundial de la Salud (OMS). 2014 Marzo; II(1).

4. Andrade Y, Coronado M, Polonia Syoc. Indagar las manifestaciones o actitudes positivas que tienen frente a la vida los adoelscentes consumidores de sustancias psicoactivas (SPA). Tesis doctoral. Hula: Universidad Nacional Abierta y a Distancia, Programa de Psicología; 2015. Report No.: UNAD/CREE - SER.

5. Pérez M, Gásquez J, Molero M. Impulsividad y consumo de alcohol y tabaco en adolescentes. Tesis doctoral. España: Universidad de Almería, Departamento de Psicología; 2015. Report No.: e - ISSN. 
6. Amaro V, Férnandez J, González M. Consumo de alcohol en niños y adolescentes. Archvos de Pediatría del Uruguay. 2016 Marzo; LXXXVII(1).

7. Ruiz H, Herrera A, Martinez A. Comportamiento adictivo de la familia como factor de riesgo de consumo de drogas en jóvenes y adolescentes adictos. Revista Cubana de Investigaciones Biomédicas. 2014; XXXIII(4).

8. The American Journal of Psichiatry. Los adolescentes son especialmente vulnerables a las adicciones. The American Journal of Psichiatry. 2013; IX(1).

9. Organización de los Estados Americanos (OEA) - Comisión Intermericana para el Control de Abuso de Drogas (CICAD). Informe del Uso de Drogas en las Américas 2015. In OAS Cataloging - in - Publication Data; 2015; Washintong. p. 33.

10. El Universo. El Universo. [Online].; 2016 [cited 2016 Enero 17. Available from: http://www.eluniverso.com/noticias/2016/04/04/nota/5504028/46-jovenes-cree-que-drogacircula-su-plantel.

11. Consejo Nacional de Control de Sustancias Estupefacientes y Psicotrópicas (CONSEP). Cuarta encuesta nacional sobre el uso de drogas en estudiantes de 12 a 17 años. 2012. Porcentajes del uso de drogas en la capital de Manabí perteneciente al país de Ecuador.

12. Organización de los Estados Americano. ¿Como funcionan las drogas? El problema de las drogas en las Américas. 2013; XXIV(4).

13. (OEA), Organización de los Estados Americanos. Las Drogas y sus efectos. El problema de las drogas en las Américas. 2013; XXIV(4).

14. Botvin G. Entrenamiento en habilidades para la vida y prevención del consumo de drogas en adoelscentes: consideraciones teorícas y hallazgos científicos. Psicología Conductual. 2014; III(3).

15. Organización de los Estados Americanos (OEA). Factores de riesgo. El impacto del consumo de drogas en la salud. 2013; XXIV(3). 
Rev. SINAPSIS, Edición № 11, Vol. 2, Diciembre 2017

ISSN $1390-9770$

Factores de riesgo para la adicción en los adolescentes www.itsup.edu.ec/myjournal 